\title{
ENERGY TRAPPING PROCESSES IN
} AROMATIC CRYSTALS

\author{
H. C. WOLF and K. W. BenZ \\ 3 Physikalisches Institut der Universität Stuttgart, \\ 7 Stuttgart 1, Azenbergstrasse 12, Germany
}

\begin{abstract}
Excitons in ideal aromatic crystals are delocalized. So far no experimental evidence for self trapping of excitons is known. Trapping of energy is possible in mixed crystals or in crystals containing defects. Disturbed exciton states due to the presence of foreign molecules or due to structural defects (the so-called $X$-traps) are the most characteristic type of defects in aromatic crystals. Some spectroscopic experimental data on $X$-traps are collected and discussed. The most sensitive method for detecting traps is that of sensitized delayed fluorescence. The kinetics of sensitized delayed fluorescence is discussed and compared with that of prompt fluorescence. Trap concentrations as low as $10^{-10} \mathrm{~mol} / \mathrm{mol}$ can be detected using delayed fluorescence.
\end{abstract}

\section{THE IDEAL CRYSTAL}

The energy levels of an ideal aromatic crystal, like naphthalene or anthracene, lie in bands: valence bands, exciton bands and conduction bands. Energy is completely delocalized in the ideal crystal.

The time scale for transitions is determined by the electronic structure of these bands, and by the transition matrix elements. Transitions between these crystal states are:

Absorption from the ground state $S_{\mathrm{o}}$ to excited states $S_{\mathrm{i}}$.

Fluorescence from the lowest excited singlet state $S_{1}$ to $S_{0}$. The lifetime of $S_{1}$ is typically between 5 and $500 \mathrm{nsec}$.

Internal conversion between different excited $S$ states or triplet $(T)$ states. These processes occur in less than $0.1 \mathrm{nsec}$.

Intersystem crossing between the singlet and the triplet manifold. The typical time constants are 100-1000 nsec.

Phosphorescence from the lowest excited triplet state $T_{1}$ to the ground state $S_{0}$. The lifetime of $T_{1}$ is typically between 1 and $10^{4} \mathrm{msec}$ and is determined by radiationless processes.

Energy storage for times longer than $0.5 \mu \mathrm{sec}$ is possible only in the $T_{1}$ band. Energy relaxation between the energy bands (internal conversion) is due to exciton-phonon interaction. Trapping processes, which are able to localize energy at specific sites in the perfect crystal are unknown so far. There is no experimental evidence for the self trapped exciton, which was first discussed by Frenkel ${ }^{1}$, and later by Sidman ${ }^{2}$. 
Self trapping of an exciton is a process in which the exciton induces a lattice relaxation, which should be responsible for some energy loss, for a Stokes-shift between absorption and emission and, perhaps, for some kind of localization of the exciton. Among the well investigated crystals only in anthracene is there a Stokes-shift between the maxima of $S_{0}-S_{1}$ absorption and $S_{1}-S_{0}$ emission, even after exclusion of other traps (Figure 1$)^{3,4}$.

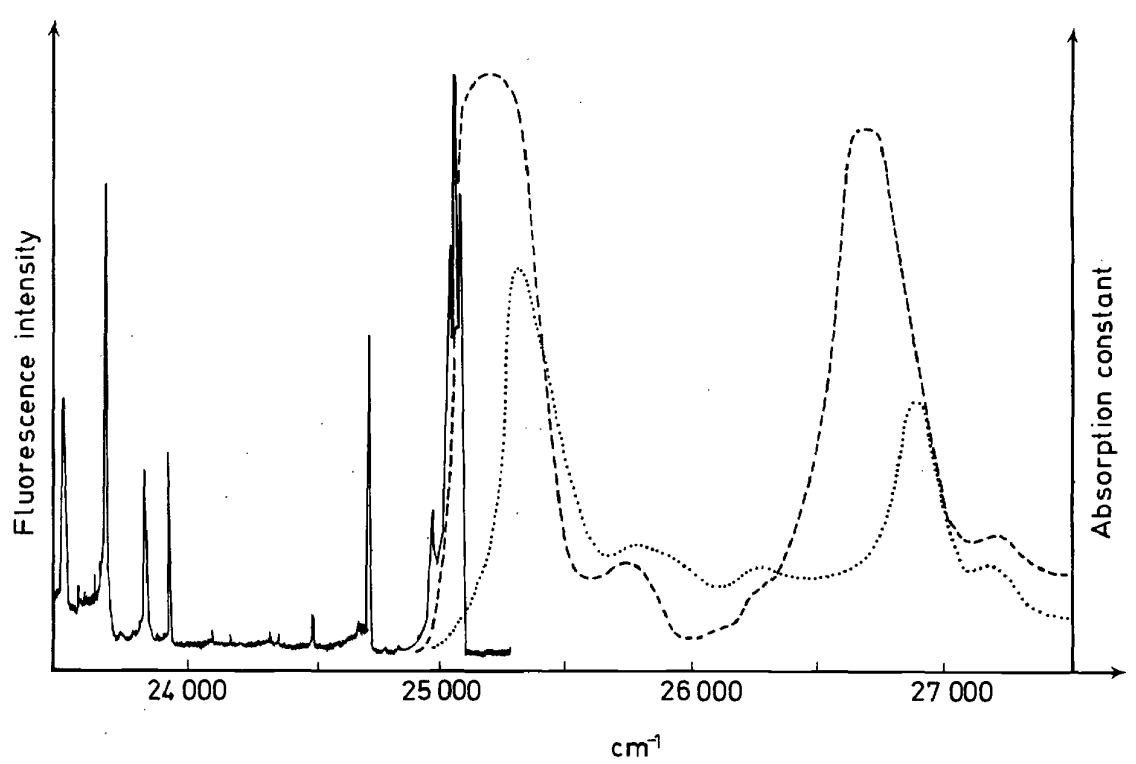

Figure 1. Absorption (right) and fluorescence (left) spectrum of crystalline anthracene near 0.0 of the lowest singlet transition. After references 3 and $4.4 .2^{\circ} \mathrm{K}$. The absorption spectrum is given parallel to axes $b(---)$ and $a(\ldots)$. The 0.0 line in emission at $25097 \mathrm{~cm}^{-1}$ is not visible due to reabsorption. The first strong emission line is the 0.0 line of the $X_{1}$-series ${ }^{4}$

But this Stokes-shift gives no evidence for exciton self trapping. The shift has been explained by the specific exciton band structure in anthracene ${ }^{4}$ and the $k$-selection rule. An alternative explanation makes use of special surface states ${ }^{5}$.

In conclusion, the problem of traps and trapping is a problem of the real, imperfect crystal.

\section{THE REAL CRYSTAL}

For the spectroscopist, the main difference between the ideal and the real crystal is the existence of traps in the latter. Very often emission spectra are, more or less, only trap spectra because of the high sensitivity of the sensitized fluorescence.

Exciton traps are sites capable of holding energy that, otherwise, propagates through the lattice. Traps are localized, non-periodic states in the crystal. Their time scale is determined by the specific electronic structure of the trap. 
Traps change the spectral energy distribution in spectra, especially in fluorescence. Traps change the time dependence of electronic population and depopulation processes in the crystal.

Traps are interesting:

as probes for measuring intrinsic properties of the host crystal, for instance the phonon structure of the host crystal from the phonon wings, which accompany guest fluorescence lines in mixed crystals ${ }^{6}$.

in themselves, because they are responsible for electronic and optical properties of the mixed or real crystal, which are different from those of the pure or ideal host crystal.

In the following two specific topics are discussed in detail: the so called $X$-traps, and the kinetics of trap-induced delayed fluorescence.

\section{DIFFERENT KINDS OF TRAPS}

In organic crystals, three types of traps have been observed and discussed so far:

1. Guest molecules, like the oldest example, tetracene in anthracene. The guest molecule can be identified by its spectral properties, which are different from those of the host crystal. Guest molecules are traps, if their $S_{1}$ state is lower than the host crystal $S_{1}$ exciton band. If these guest molecules are deep traps $(\Delta E \gg k T)$, sensitized fluorescence is observed.

2. Self trapping. This process has been discussed in section I.

3. Disturbed exciton states in the crystal, X-traps. These $X$-traps are very characteristic defects in organic crystals. The name $X$-series has been coined for vibronic series in the luminescence spectra of crystals which are identical with the intrinsic host emission, but red shifted by a certain amount of energy ${ }^{7}$. This is the emission from disturbed regions of the crystal.

The perturbation can be due to the presence of foreign molecules (impurities

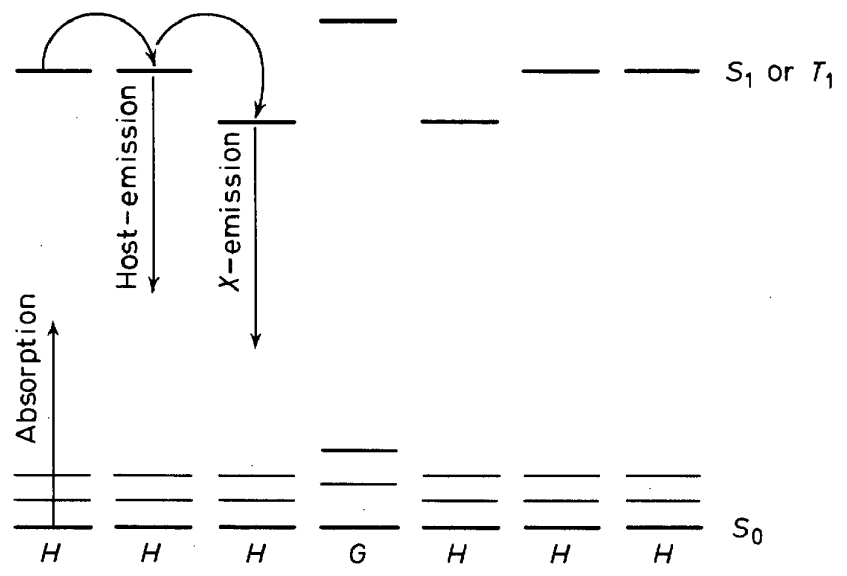

Figure 2. Energy level scheme of a host $(H)$ crystal with guest $(G)$ molecules, which induce $X$-traps. For simplification, the host exciton band is approximated by the individual molecule energy levels 
or guest molecules) in the matrix, which cannot act as traps themselves, because the energy of their lowest $S_{1}$ state is higher than the bottom of the host $S_{1}$ band. But host molecules around these impurities are shifted in energy and can act as energy traps. A schematic energy level diagram for $X$-traps is shown in Figure 2. These impurity-induced $X$-traps can be removed partially or completely by crystal purification.

The second possible reason for $X$-series can be structural defects or dislocations in the lattice. These $X$-traps are insensitive towards purification processes, but they can be removed by annealing.

It is impossible to understand most of the spectroscopic solid-state properties of organic crystals without taking into account these $X$-traps. They are the analogues of the so called $\alpha$ and $\beta$-bands in alkali halides ${ }^{8}$, which have been well known for nearly forty years.

It is important to notice that the $X$-traps, which have been found so far, are always relatively shallow traps. At room temperature they are more or less ineffective.

\section{EXAMPLES OF X-TRAPS IN OPTICAL SPECTRA}

In this section are collected some experimental data on $X$-traps in different crystals, and identified by different methods.

In the fluorescence spectrum of naphthalene crystals of high purity, 4 main series of lines have been identified ${ }^{7}$ : one of them, with 0.0 at $31474 \mathrm{~cm}^{-1}$, is the emission originating from the exciton band $S_{1}$. The other three, with 0.0 at 31444,31418 and 31395 , are emission series originating from naphthalene molecules with an $S_{1}$-level $\Delta E=30,56$ and $79 \mathrm{~cm}^{-1}$, respectively, below the bottom of the naphthalene exciton band.

The perturber which induces the $30 \mathrm{~cm}^{-1} X$-trap has been identified as thionaphthene. The other perturbers are impurities $X$, unidentified so far. This is the reason for the name $X$-trap. All the $X$-series have the vibronic progressions characteristic of the naphthalene molecule. The $X$-series are identical in energy with the naphthalene series, but shifted to lower energy by $\Delta E$. The lines of the $X$-series are much sharper than the lines in the host series.

In contrast to these impurity-induced $X$-traps, $\beta$-methyl-naphthalene in naphthalene is a real trap, with 0.0 at $31059 \mathrm{~cm}^{-1}$ and a vibronic structure of the fluorescence spectrum characteristic of the $\beta$-methyl-naphthalene molecule.

On can create additional $X$-traps by introducing structural imperfections into the crystal. In naphthalene this has been investigated using plastic deformation ${ }^{9}$. In the fluorescence spectrum of plastically deformed naphthalene crystals, one observes three different characteristic structures:

1. One sharp $X$-series, shifted $165 \mathrm{~cm}^{-1}$ to lower energies $(0.0$ at $31309 \mathrm{~cm}^{-1}$ ).

2. A continuous background (called subnaphthalene series in reference 9).

3. Emission typical of excimers with the maximum at about $24000 \mathrm{~cm}^{-1}$. These three types of spectra can be reduced in intensity by annealing. In some cases, annealing is improved by simultaneous excitation with light ${ }^{9}$. The relative intensity of the different spectra is a function of temperature, corresponding to the different trap depth. 


\section{ENERGY TRAPPING PROCESSES IN AROMATIC CRYSTALS}

The fluorescence spectrum of extremely pure anthracene crystals also contains many lines which are due to $X$-traps. In addition to the intrinsic fluorescence, originating at $25097 \mathrm{~cm}^{-1}, X$-series are observed ${ }^{10}$ with 0.0 at $25074,25030,24850$ and $24823 \mathrm{~cm}^{-1}$. Similar observations are reported by other authors ${ }^{11,12}$.

$X$-traps are present also in the triplet state $T_{1}$. It is much easier to observe them via delayed fluorescence than directly by phosphorescence spectra. In naphthalene, the following triplet state $X$-traps have been identified ${ }^{13}$ : thionaphthene introduces an $X$-trap with $\Delta E=45 \mathrm{~cm}^{-1}$, durene one at $60 \mathrm{~cm}^{-1}$, and other $X$-traps are present which have not been identified so $\mathrm{far}^{14}$. As in the singlet state, $\beta$-methyl-naphthalene is a guest molecule, not an $X$-trap, with $\Delta E=240 \mathrm{~cm}^{-1}$. In anthracene crystals, the trap ${ }^{10}$ $X_{4}$ which is $274 \mathrm{~cm}^{-1}$ below the $S_{1}$ exciton band is also an $X$-trap in the triplet state, with ${ }^{15} \Delta E=24 \mathrm{~cm}^{-1}$.

In addition to the unperturbed exciton emission of the host and $X$-series, the spectrum of delayed fluorescence in anthracene and naphthalene crystals contains a continuous background, which is due to a continuous energy distribution of traps ${ }^{14,15}$. All types of trap spectra are much more pro-

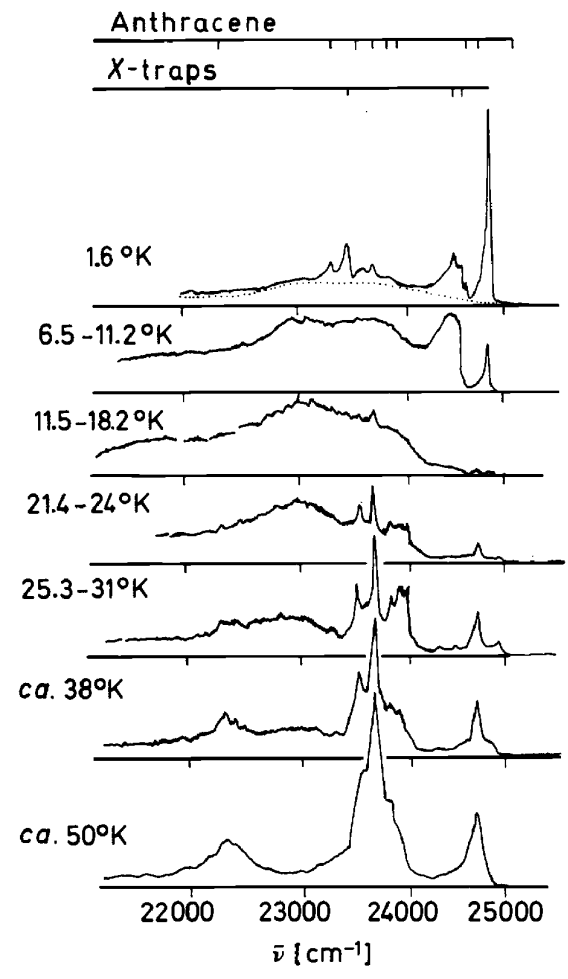

Delayed fluorescence

Anthracene
$x$-traps

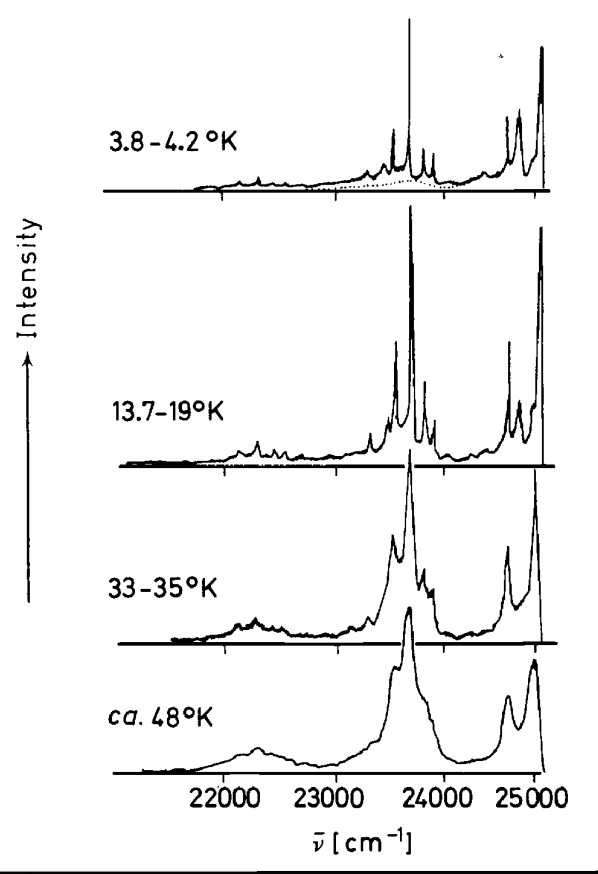

Prompt fluorescence

Figure 3. Spectrum of delayed and prompt fluorescence of very pure anthracene crystals between 1.6 and $50^{\circ} \mathrm{K}$. From reference 15 
H. C. WOLF AND K. W. BENZ

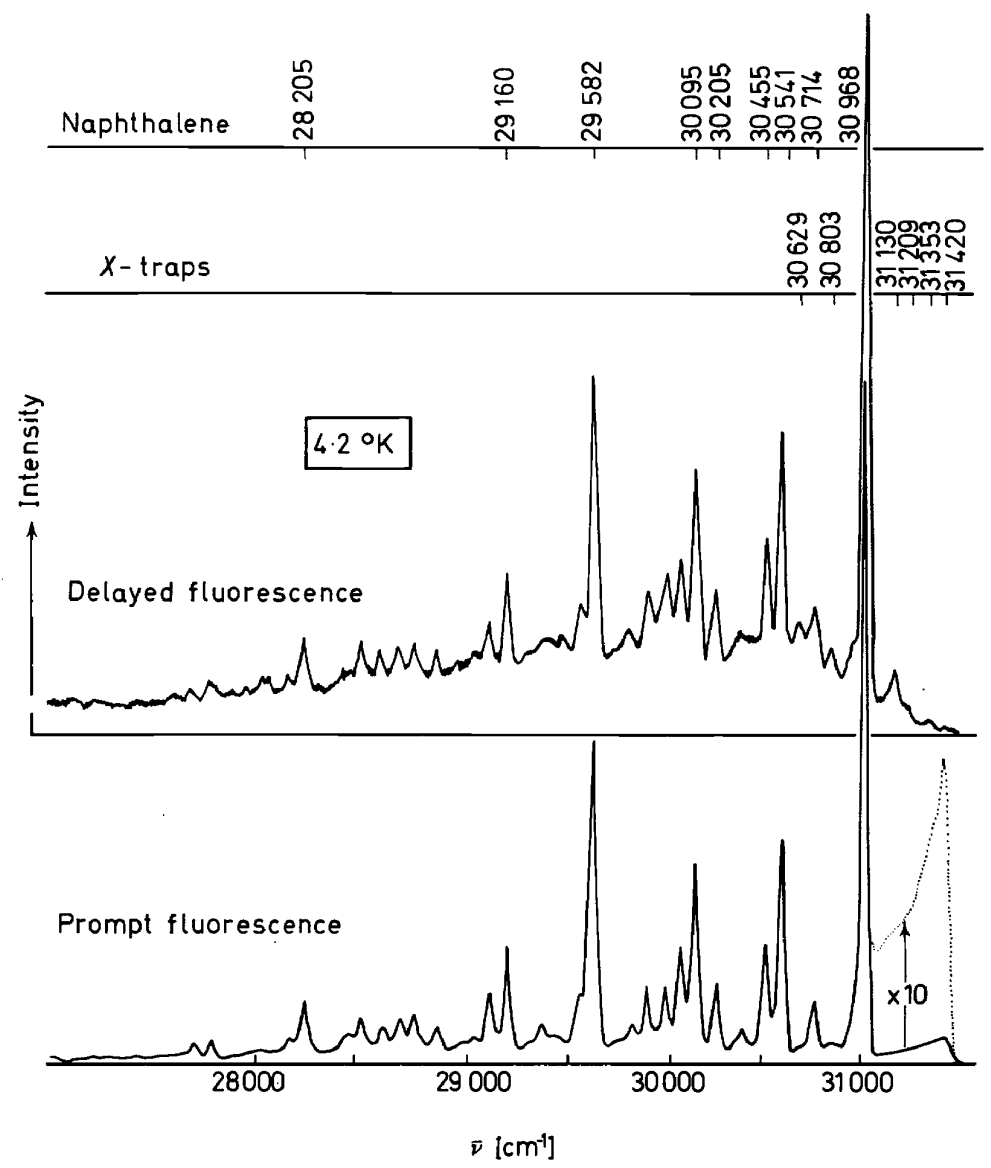

Figure 4. Spectrum of delayed and prompt fluorescence of a very pure naphthalene crystal at $4.2^{\circ} \mathrm{K}$. From reference 15

nounced in delayed fluorescence than in prompt fluorescence-an observation which will be discussed in section VI. The trap spectra disappear with increasing temperature when the traps are thermally depopulated. Figure 3 shows the temperature dependence of delayed and prompt fluorescence in pure anthracene crystals. In delayed fluorescence the trap emission is much more pronounced than in prompt fluorescence. A plot of the $X$-trap emission relative to the continuous background versus temperature (Figure 5) can be used to determine the trap depth $\Delta E$.

$X$-traps are also observable in phosphorescence. In pyrazine crystals at $4.2^{\circ} \mathrm{K}$ two $X$-series have been observed ${ }^{16}$. Azumi called them impurity induced resonance defects.

\section{V. $X$-TRAPS IN ESR SPECTRA}

$X$-traps can be identified and are important also in ESR spectra of the metastable triplet state $T_{1}$ at low temperature. A good example is quinoxaline 


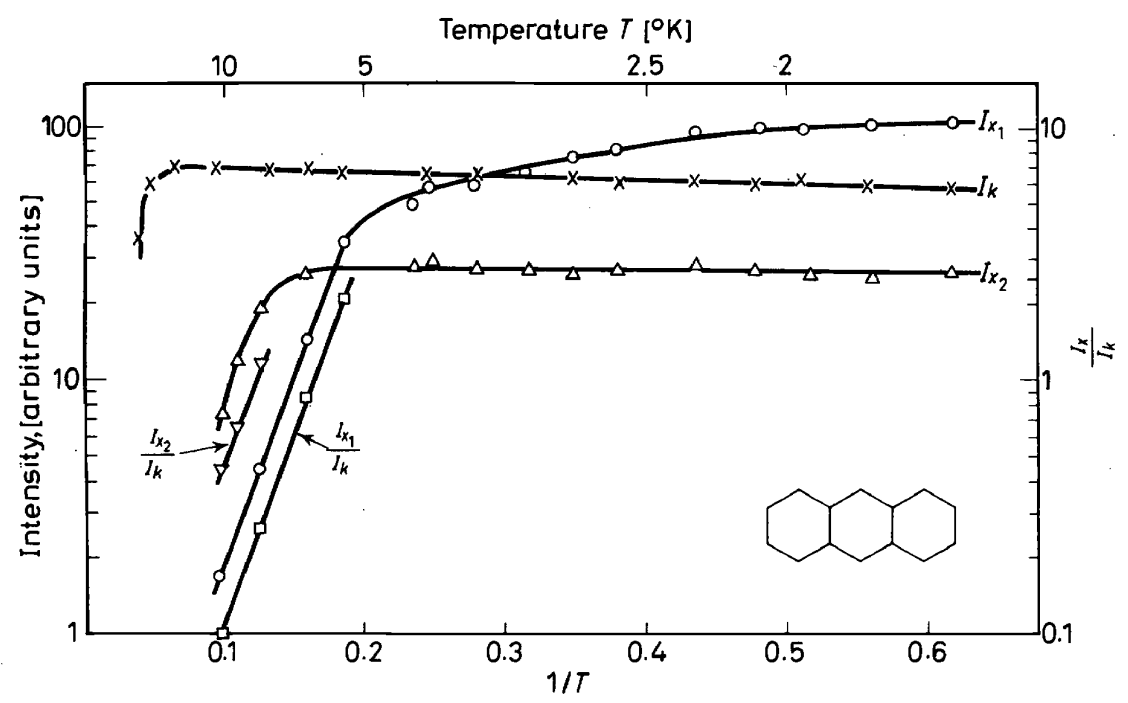

Figure 5. Temperature dependence of the intensity $I$ of the series $X_{1}, X_{2}$ and the continuum $K$ in the delayed fluorescence spectrum of anthracene crystals between 1.6 and $20^{\circ} \mathrm{K}$. Determination of the trap depth $\Delta E$. From reference 15

in a naphthalene matrix ${ }^{17}$. In a matrix of normal naphthalene $\left(\mathrm{N}-\mathrm{h}_{8}\right)$, quinoxaline introduces an $X$-trap with $\Delta E=60 \mathrm{~cm}^{-1}$. The ESR spectrum observed at low temperature $\left(<4.2^{\circ} \mathrm{K}\right.$ ) (Figure 6) is therefore due to naphthalene molecules. This is demonstrated by the hyperfine structure of the ESR spectrum. The hyperfine structure is due to the four equivalent $\alpha$ protons in the naphthalene molecule. The hyperfine structure due to the $\beta$-protons cannot be resolved. According to the angular dependence of the ESR-spectrum, the misorientation of the $X$-trap naphthalene molecule is less than $2^{\circ}$. In addition, the ESR spectrum shows no indication of any delocalization of the triplet energy. This means that the $X$-trap is probably only one very slightly disturbed naphthalene molecule in the vicinity of the quinoxaline molecule. With increasing temperature the ESR signal vanishes due to thermal detrapping (Figure 7).

In contrast to $\mathrm{N}-\mathrm{h}_{8}$, quinoxaline in perdeutero-naphthalene is a real trap. The ESR spectrum of $\mathrm{N}-\mathrm{d}_{8}$ crystals doped with quinoxaline is therefore a quinoxaline spectrum. The hyperfine structure is typical of two equivalent $\alpha$ protons and four equivalent $\beta$-protons (Figure 6).

ESR and ENDOR spectroscopy is a powerful method for getting very detailed information on the structure of $X$-traps. Other $X$-traps which have been identified, using ESR-spectroscopy, are due to quinazoline and thionaphthene in naphthalene and dibenzothiophene and carbazole in fluorene ${ }^{18}$.

Theoretical calculations on the electronic structure of disturbed exciton states were first published by Merrified ${ }^{19}$. A very detailed comprehensive review is given by Rice and Jortner ${ }^{20}$. Many new experimental data have been collected in the meantime. It seems worthwhile to use these data as a 


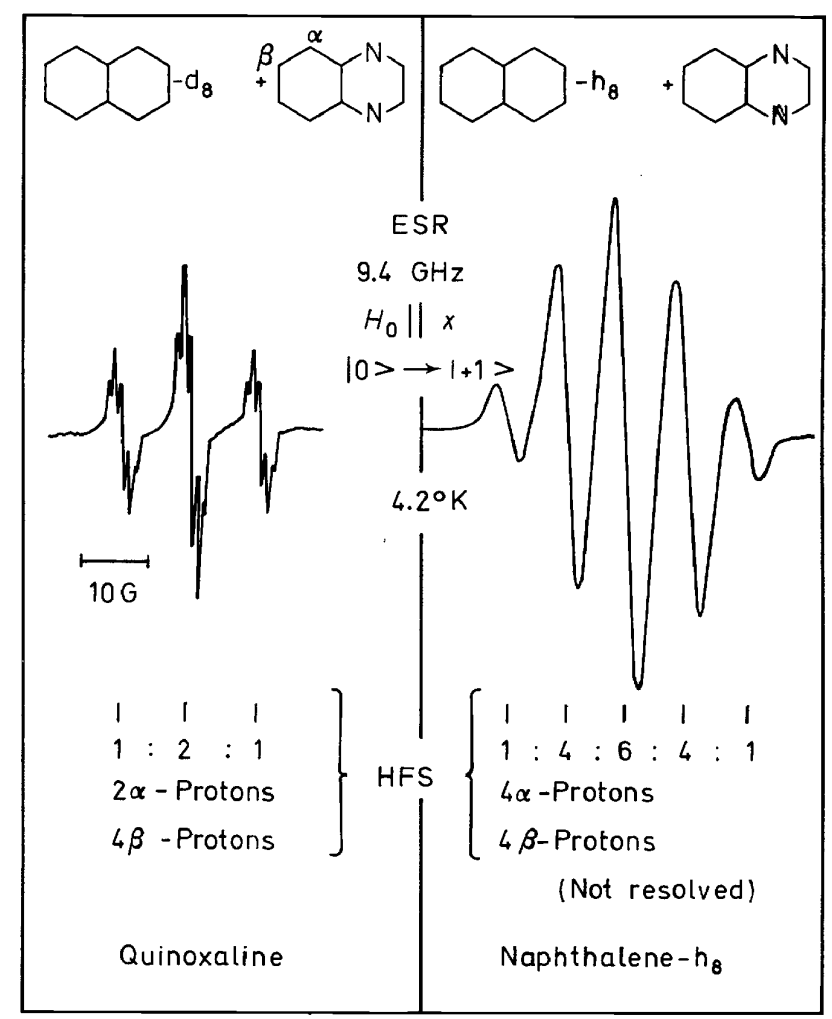

Figure 6. Hyperfine structure of one ESR-line ( $\Delta m=1$ transition) in the ESR spectrum of the triplet state of quinoxaline in perdeutero-naphthalene (left), and of the $X$-trap which is induced by quinoxaline in nahpthalene (right). The quinoxaline concentration was the same in both crystals. The hyperfine structure is characteristic of quinoxaline (left) and of naphthalene (right).

(This figure was kindly supplied by M. Schwoerer)

basis for more detailed theoretical work, which could help us to get a better understanding of the nature of these defects in molecular crystals. In semiconducting materials, like silicon and germanium, similar disturbed exciton states are known. An empirical rule has been proposed ${ }^{21}$, which correlates the trap depth and the physical nature of the perturber.

In a series of recent papers, Jortner $e t a l .^{22}$ have treated the $X$-trap problem theoretically in a very detailed manner. They have shown that $X$-trapping is due to the solvent shift terms. An $X$-trap host molecule is just a host molecule in a different environment. It seems worthwhile to apply these calculations to the experimental data collected above.

In Table 1 experimental data on $X$-traps in naphthalene and anthracene crystals are collected.

\section{KINETICS OF TRAPPING PROCESSES}

Trapping processes are responsible for the well known phenomenon of 


\section{ENERGY TRAPPING PROCESSES IN AROMATIC CRYSTALS}

Table 1. Some $X$-traps in naphthalene and anthracene and their trap depths, $\Delta E$, in the singlet and in the triplet state

\begin{tabular}{|c|c|c|c|c|}
\hline Matrix & Perturber & $\underset{\mathrm{cm}^{-1}}{\Delta E \text { in } S_{1}}$ & $\begin{array}{c}\Delta E \text { in } T_{1} \\
\mathrm{~cm}^{-1}\end{array}$ & References \\
\hline \multicolumn{5}{|l|}{ Naphthalene } \\
\hline & thionaphthene & 30 & 45 & 7,13 \\
\hline & unknown impurity & 56 & & 7 \\
\hline & unknown impurity & 79 & & 7 \\
\hline & structural defect & 165 & & 9 \\
\hline & continuum & continuous & & 9 \\
\hline & durene & & 60 & 13 \\
\hline & quinoxaline & & ca. 60 & 17 \\
\hline & unknown & 144 & & 14 \\
\hline & unknown & 268 & 23 & 14 \\
\hline & unknown & 346 & 32 & 14 \\
\hline \multicolumn{5}{|l|}{ Anthracene } \\
\hline & unknown defects & 23 & & 10 \\
\hline & or impurities & 67 & & 10 \\
\hline & & 247 & & 10 \\
\hline & & 274 & 24 & 10,15 \\
\hline & continuum & continuous & & \\
\hline
\end{tabular}

sensitized fluorescence, where low concentrations of impurity molecules are able to convert the host fluorescence into guest (impurity) emission. The delayed fluoresence is even more sensitive towards impurities, because of the effectiveness of energy transfer, and sensitized delayed fluorescence is a method for detecting impurities or traps in concentrations a hundred times lower than is sensitized prompt fluorescence.

This is demonstrated by Figures 8 and 9. Figure 8 shows the fluorescence spectrum of a mixed crystal naphthalene with $2 \times 10^{-7}$ parts of anthracene. Whereas in prompt fluorescence the anthracene concentration is too low to convert much of the naphthalene emission into anthracene fluorescence and anthracene emission is almost absent, in delayed fluorescence the anthracene emission is more intense than that of naphthalene. Energy transfer can be measured quantitatively by determining the quantum ratio $Q_{G} / Q_{H}$ (guest quanta divided by host quanta) as a function of guest concentration. This quantum ratio is, according to Figure 9, 100 times higher in delayed fluorescence than in prompt fluorescence. Also Figures 3 and 4 demonstrate the much higher sensitivity of delayed fluorescence than of prompt fluorescence towards impurities and traps.

In this section, the effectiveness of delayed sensitized fluorescence is calculated and compared with that of sensitized prompt fluorescence.

The kinetics of population and depopulation of traps, which can be guest molecules or $X$-traps, is described in the following general scheme (Figure 10):

1. Excitons are created by absorption of light of intensity $I$ with absorption coefficient $\alpha_{S}$ or $\alpha_{T}$. Exciton bands $S_{1}$ or $T_{1}$ are populated by internal conversion and intersystem crossing.

2. Excitons can decay with emission of a photon, radiatively, into the ground state $S_{\mathrm{o}}$ with a rate constant $k_{H}^{*}$ for singlets and $\beta_{H}^{*}$ for triplets. 
H. C. WOLF AND K. W. BENZ

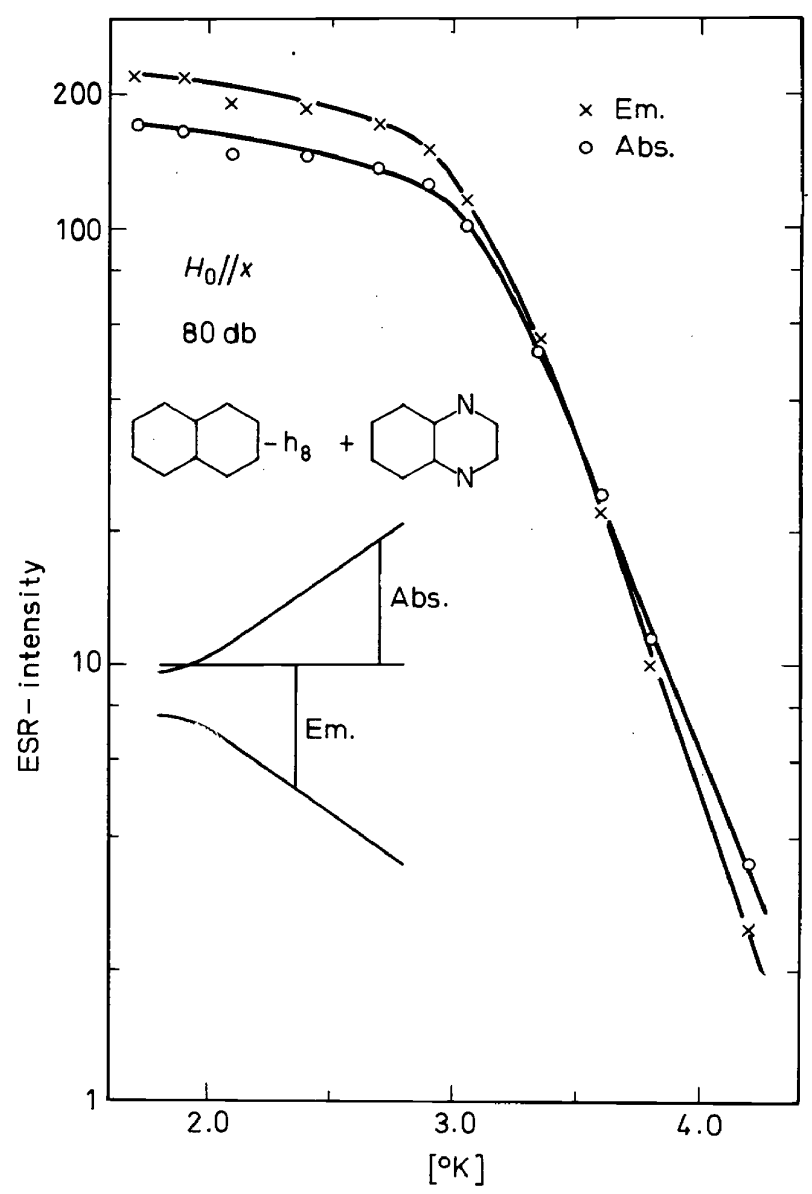

Figure 7. Temperature dependence of the intensity of the two ESR lines of the $X$-trap induced in naphthalene by quinoxaline. One of the two lines is emissive due to optical spin polarization.

(This figure was kindly supplied by M. Schwoerer)

3. Radiationless processes are competitive, with rate constants $k_{H}^{\prime}$ and $\beta_{H}^{\prime} ; k=k^{\prime}+k^{*}, \beta=\beta^{\prime}+\beta^{*}$.

4. Excitons can relax into traps, which are $\Delta E$ lower in energy with a rate constant $k_{H G}$ and $\beta_{H G}$.

5. Traps can be thermally depopulated back into the exciton band, if the temperature is high enough.

6. Traps ca. he depopulated by emission of a photon radiatively into the ground state $S_{0}$, rate constant $k_{G}^{*}$.

7. Traps can be depopulated nonradiatively $k_{G}^{\prime}$.

8. Triplet traps can be depopulated by triplet-triplet annihilation, annihilation coefficient $\gamma_{G}$.

9. Triplet annihilation is also effective in the $T_{1}$ exciton band, without traps, annihilation coefficient $\gamma$. 


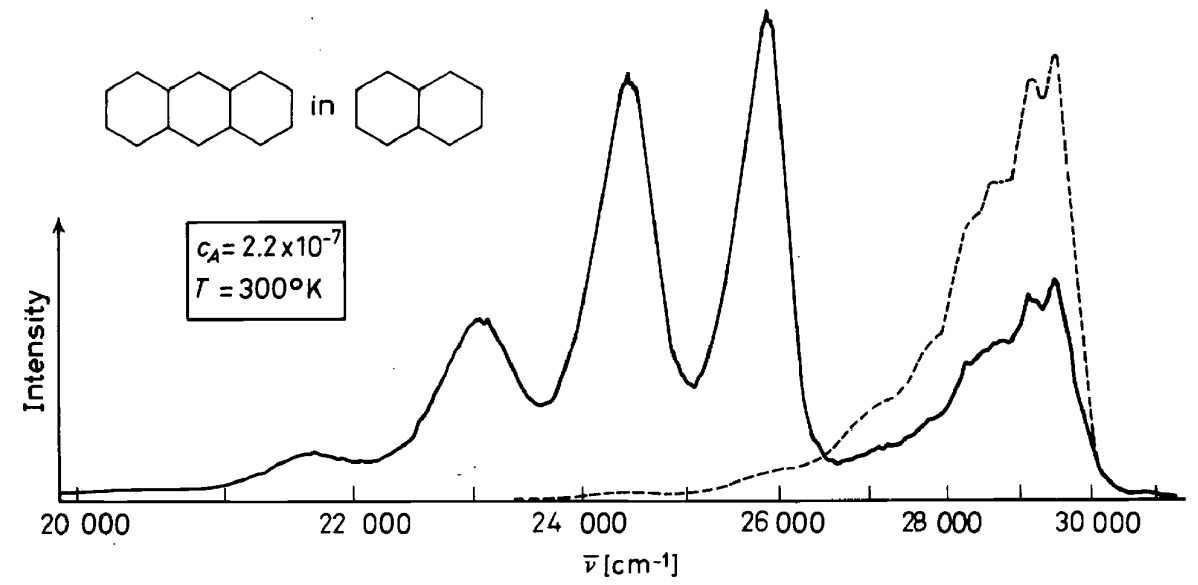

Figure 8. Spectrum of prompt (broken line) and delayed (full line) fluorescence in a naphthalene crystal containing $2.2 \times 10^{-7}$ parts of anthracene, at room temperature. Anthracene emission is below $26500 \mathrm{~cm}^{-1}$. From reference 15

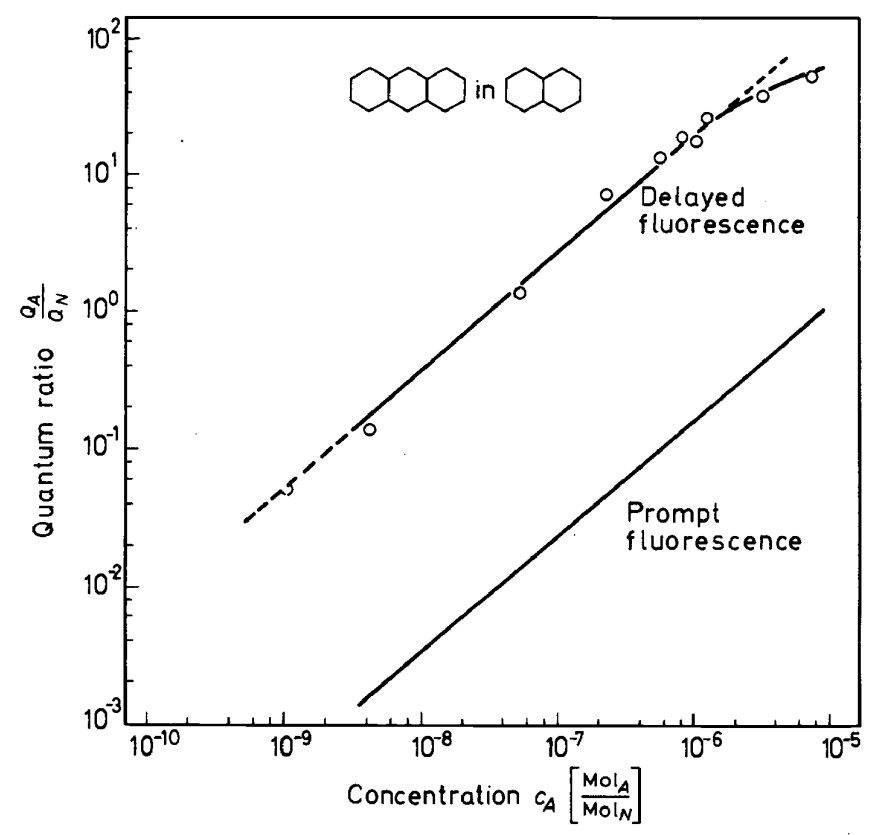

Figure 9. Quantum ratio $Q_{A} / Q_{N}$ in naphthalene crystals with different anthracene concentrations, delayed and prompt fluorescence. The concentration was measured by absorption. From references 14 and 15 


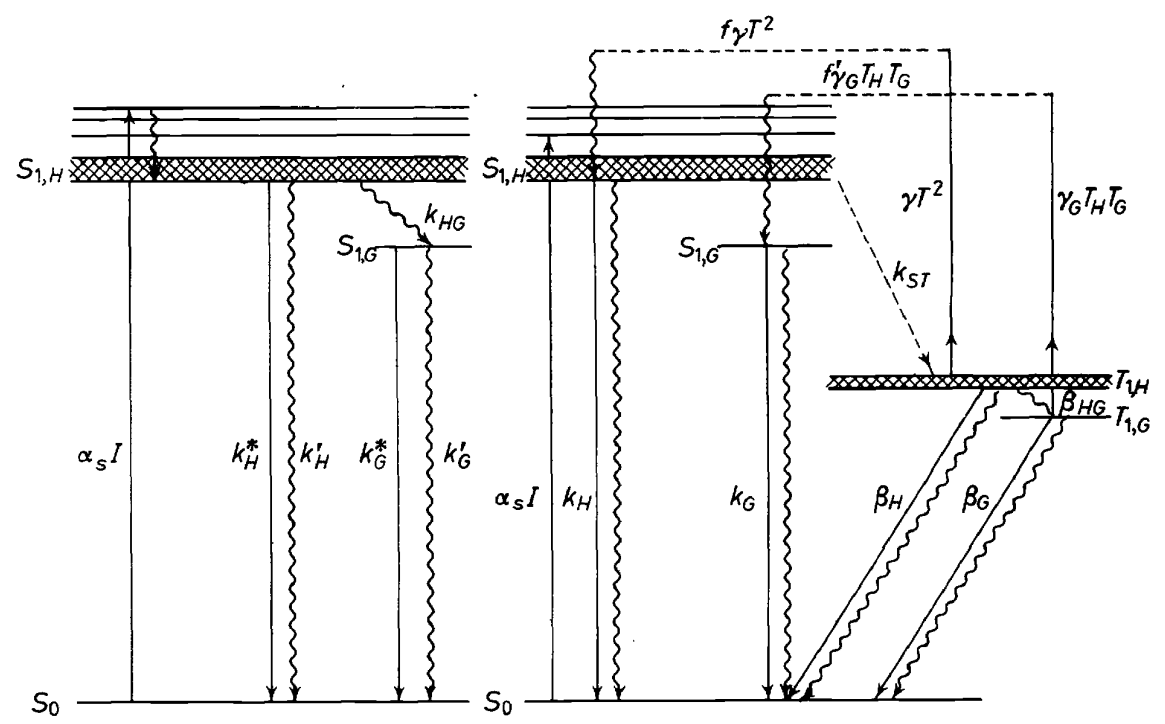

Figure 10. Kinetics of sensitized prompt fluorescence (left) and sensitized delayed fluorescence (right) for a host-guest system

The process which determines the effectiveness of traps is mainly process 4 , the exciton capture by traps. During their lifetimes excitons scan the crystal for traps. The concentration of traps, the exciton lifetime and the diffusion rate of the exciton determine, therefore, the capture probability.

Many different models for exciton motion have been discussed in the past. It seems that the very simple hopping model is a quite adequate description ${ }^{23}$, at least as long as energy transfer is concerned. In this model the exciton jumps statistically from molecule to molecule in the lattice with a characteristic hopping time $t_{h}$. This time is related to the diffusion coefficient $D$ by an expression of the form ${ }^{23} t_{h}=\pi D R N_{H} ; R=$ defect radius, $N_{H}=$ number of host molecules per $\mathrm{cm}^{3}$. The number of lattice sites visited during the lifetime is given by the quotient lifetime divided by hopping time (if one neglects the probability of visiting the same site more than once) ${ }^{24}$. This model has been discussed in great detail by Suna recently ${ }^{25}$. In prompt fluorescence one measures the singlet traps which are populated by the scanning exciton, in delayed fluorescence the triplet traps.

The quantum ratio of guest to host emission is calculated in the following way (Figure 10):

\section{A. Prompt fluorescence}

The rate equations for host and guest singlet excitons $S_{H}$ and $S_{G}$ (number of excited states per volume) are in the deep trap limit $(\Delta E \gg k T)$, neglecting intersystem crossing into the triplet state,

$$
\frac{\mathrm{d} S_{H}}{\mathrm{~d} t}=\alpha_{S} I-k_{H} S_{H}-k_{H G} S_{H}
$$




$$
\frac{\mathrm{d} S_{G}}{\mathrm{~d} t}=k_{H G} S_{H}-k_{G} S_{G}
$$

The quantum flux $Q_{H}, Q_{G}$ (number of quanta per volume and sec) is given by

$$
\begin{aligned}
& Q_{H}=k_{H}^{*} S_{H} \\
& Q_{G}=k_{G}^{*} S_{G}
\end{aligned}
$$

In the stationary case, $d S / \mathrm{d} t=0$, one gets from (2)

$$
\frac{S_{G}}{S_{H}}=\frac{k_{H G}}{k_{G}}
$$

and finally the quantum ratio

$$
\left(\frac{Q_{G}}{Q_{H}}\right)_{\text {prompt fluorescence }}=\frac{k_{G}^{*}}{k_{H}^{*}} \times \frac{k_{H G}}{k_{G}}
$$

(5) can also be written in the form

$$
\begin{aligned}
\left(\frac{Q_{G}}{Q_{H}}\right) P F & =\eta_{G} \frac{k_{H G}}{k_{H}^{*}} \\
\eta_{G} & =\text { quantum yield of the guest fluorescence }
\end{aligned}
$$

In the hopping model, the simplest assumption for the transfer rate constant $k_{H G}$ is

$$
\begin{aligned}
k_{H G} & =\frac{c_{G}}{t_{h}}, \text { singlet } \\
c_{G} & =\text { guest concentration in } \mathrm{mol} / \mathrm{mol} \\
t_{h} & =\text { hopping time }
\end{aligned}
$$

With (5) and (6) one gets as quantum ratio

$$
\begin{aligned}
&\left(\frac{Q_{G}}{Q_{H}}\right)_{\text {prompt fluorescence }}=\frac{\eta_{G} c_{G}}{k_{H}^{*} t_{h}, \text { singlet }}=K_{\text {singlet }} \times c_{G} \\
& K= \text { transfer constant as defined in } \\
& \text { reference } 23 .
\end{aligned}
$$

Equation (6) is based on the following simplifications:

1. It is assumed that transfer of excitation is by hopping from molecule to the nearest neighbour molecule, and that the capture cross section is the same for host and guest molecules. The validity of this assumption is questioned by new experiments of Baessler et al. ${ }^{32}$.

2. It is assumed that the probability for the hopping exciton to meet a guest molecule is proportional to the guest concentration. Due to the possibility that the excitation is able to visit one lattice site more than once, this assumption is valid only at low concentrations ${ }^{24}$. 
Equation (7) has been derived and verified in the system anthracene/ tetracene ${ }^{26}$. In this system, the measured transfer constant $K_{\text {singlet }}$ in the equation $Q_{G} / Q_{H}=K \times c_{G}^{p}$, is $(6 \pm 3) \times 10^{4}$, with the exponent $p=0.8 \pm 0.2$. $K$-values in other systems have the same order of magnitude ${ }^{23}$. At a guest concentration of $c_{G}=10^{-7}$, the quantum ratio is 0.01 . Therefore it is possible to detect traps and impurities using sensitized fluorescence at concentrations down to $10^{-7}-10^{-8}$.

Using equation (7), the hopping time $t_{h}$ can be calculated. For the system anthracene in naphthalene, the following value has been measured:

$$
K_{\text {singlet }}=10^{5} \text { (Figure 9) }
$$

Taking $\eta_{G}$ (anthracene in naphthalene) $=1$ and $k_{H}^{*}$ (naphthalene, room temperature) $=2 \times 10^{6} \mathrm{sec}^{-1}$, one gets for naphthalene

$$
t_{h, \text { singlet }}=5 \times 10^{-12} \sec ( \pm 50 \%)
$$

Equations (1)-(7) are valid for a two component system, host and guest. If there are other traps present with a trap depth comparable to $k T$, then the apparent quantum ratio is a function of temperature ${ }^{23,26}$. By this freezing of energy transfer one can measure trap concentrations down to approximately $10^{-7}$.

\section{B. Delayed fluorescence}

Again we discuss only a two component system (guest and host), and we assume homogeneous excitation. If one excites into the singlet state, the following rate equations describe the time dependence of concentration of triplet excitons, $T_{H}$ and $T_{G}$ (Figure 10):

$$
\begin{aligned}
\frac{\mathrm{d} T_{H}}{\mathrm{~d} t} & =\eta_{S T} \alpha_{S} I-\beta_{H} T_{H}-\beta_{H G} T_{H}-\gamma_{G} T_{H} T_{G}-\gamma T_{H}^{2} \\
\eta_{S T} & =\text { quantum yield for intersystem crossing } \\
\frac{\mathrm{d} T_{G}}{\mathrm{~d} t} & =\beta_{H G} T_{H}-\beta_{G} T_{G}-\gamma_{G} T_{H} T_{G}
\end{aligned}
$$

If one excites directly into the triplet state, one has to replace the expression $\eta_{S T} \alpha_{S} I$ in (11) by $\alpha_{T} I$.

At low concentration, delayed host fluorescence is entirely due to host/host annihilation, and delayed guest fluorescence entirely due to host/guest annihilation. Therefore the rate equations for singlet excitons created by triplet-triplet annihilation are:

$$
\begin{aligned}
& \frac{\mathrm{d} S_{H}}{\mathrm{~d} t}=\frac{1}{2} f \gamma T_{H}^{2}-k_{H} S_{H} \\
& \frac{\mathrm{d} S_{G}}{\mathrm{~d} t}=\frac{1}{2} f^{\prime} \gamma_{G} T_{H} T_{G}-k_{G} S_{G}
\end{aligned}
$$

$f$ and $f^{\prime}$ are the fraction of triplet-triplet annihilation processes which result in singlet states $S_{1} . f$ and $f^{\prime}$ are near ${ }^{27} 0.4$. 
In the stationary case,

$$
\frac{\mathrm{d} T_{\mathrm{H}}}{\mathrm{d} t}=\frac{\mathrm{d} T_{G}}{\mathrm{~d} t}=\frac{\mathrm{d} S_{H}}{\mathrm{~d} t}=\frac{\mathrm{d} S_{G}}{\mathrm{~d} t}=0 .
$$

Saturation of traps is neglected. Using equations (3), (4), (13) and (14), one gets the quantum ratio:

$$
\left(\frac{Q_{G}}{Q_{H}}\right)_{\text {Delayed fluorescence }}=\frac{\eta_{G}}{\eta_{H}} \times \frac{f^{\prime} \gamma_{G}}{f \gamma} \times \frac{T_{G}}{T_{H}}
$$

The triplet concentrations $T_{G}$ and $T_{H}$ are a function of the intensity $I$ of the exciting light. At low excitation intensities (or low stationary triplet concentrations), the triplet lifetime is determined by monomolecular decay, at high excitation intensities by bimolecular annihilation. The two limiting cases have to be discussed separately.

a. Weak excitation, $\beta_{G} T_{G} \gg \gamma T_{G} T_{H}$

Equation (12) transforms into

$$
\frac{T_{G}}{T_{H}}=\frac{\beta_{H G}}{\beta_{G}}
$$

Equation (12a) is completely analogous to the singlet state equation (2a), and the quantum ratio is independent of $I$ :

$$
\frac{Q_{G}}{Q_{H}}=\frac{\eta_{G}}{\eta_{H}} \frac{f^{\prime} \gamma_{G}}{f \gamma} \frac{\beta_{H G}}{\beta_{G}}
$$

In the hopping model, the transfer rate is given by

$$
\beta_{H G}=\frac{c_{G}}{t_{h, \text { triplet }}}
$$

and finally one gets the quantum ratio

$$
\begin{aligned}
\left(\frac{Q_{G}}{Q_{H}}\right)_{\begin{array}{c}
\text { Delayed fluorescence, } \\
\text { weak excitation }
\end{array}} & =\frac{\eta_{G} f^{\prime} \gamma_{G}}{\eta_{H}} \frac{c_{G}}{f \gamma} \frac{\beta_{G} t_{h, \text { triplet }}}{} \\
& =K_{\text {triplet }} \times c_{G}
\end{aligned}
$$

Comparing equations (7) and (17) one realises immediately that the ratio of the transfer constants $K_{\text {triple }} / K_{\text {singlet }}$ is determined by the ratio

$$
\frac{k_{H}^{*} t_{h, \text { singlet }}}{\beta_{G} t_{h, \text { triplet }}}
$$

Now the experimental results (Figure 9) are compared with equation (17). In the system anthracene in naphthalene, the experimentally determined transfer constant is roughly

$$
K_{\text {triplet }}=3 \times 10^{7} \text { (Figure 9) }
$$


We use the following approximate numerical values:

$\eta_{G}=1^{28}, \eta_{H}=0.2^{28}, f=0.4^{27}, \gamma=3.5 \times 10^{-12} \mathrm{~cm}^{3} \mathrm{sec}^{-114}$, and we assume without experimental proof that $f=f^{\prime}$ and $\gamma=\gamma_{G} ; \beta_{G}=300 \mathrm{sec}^{-114}$. So we get the triplet hopping time $t_{h}$ :

$$
t_{h}=5 \times 10^{-11} \sec ( \pm 50 \%)
$$

This value is consistent with results from $\mathrm{ESR}^{29}$ and $\mathrm{NMR}^{30}$ measurements.

Now we are able to discuss the limiting sensitivity of sensitized delayed fluorescence for detecting impurities. Since the transfer constant $K=3 \times 10^{7}$, the quantum ratio equation (17) is 0.01 at $c_{G}=3 \times 10^{-10}$. Consequently, using delayed fluorescence, one is able to detect anthracene in naphthalene at concentrations as low as $10^{-10}$, more than two orders of magnitude lower than using prompt fluorescence. This is mainly due to the long lifetime of the triplet traps, $\beta_{G}$ in equation (17), since the hopping time $t_{h}$ is 10 times longer for triplet excitons than for singlets. Similar conclusions have been derived for the mixed system anthracene in phenanthrene ${ }^{31}$.

ק. Strong excitation, $\beta_{G} T_{G} \ll \gamma_{G} T_{G} T_{H}$

From equation (12) one gets

$$
T_{G}=\frac{\beta_{H G}}{\gamma_{G}}
$$

and from (15)

$$
\left(\frac{Q_{G}}{Q_{H}}\right)_{\substack{\text { Delayed fluorescence } \\ \text { strong excitation }}}=\frac{\eta_{G}}{\eta_{H}} \frac{f^{\prime}}{f \gamma} \frac{\beta_{H G}}{T_{H}}
$$

In this case, the quantum ratio is dependent on the intensity of excitation $I$. In the limiting case of high intensity and low guest concentration, $T_{H} \simeq(\alpha I / \gamma)^{\frac{1}{2}}$. Therefore the quantum ratio is proportional to $I^{-\frac{1}{2}}$. This is demonstrated in Figure 11.

In multi-component systems the intensity relations are much more complex because there is competition between different traps. The extremely high sensitivity of sensitized delayed fluorescence is valid apparently only at not too low temperatures, where the $X$-traps and all kinds of shallow traps are no longer effective. In the system naphthalene $+\beta$-methyl-naphthalene, the quantum ratios for prompt and for delayed fluorescence at $4.2^{\circ} \mathrm{K}$ are nearly equal ${ }^{14}$. This is due to the presence of $X$-traps in naphthalene, and to the introduction of additional shallow traps when doping naphthalene with $\beta$-methylnaphthalene. These shallow traps are responsible for the very strong temperature dependence of delayed fluorescence intensity below $100^{\circ} \mathrm{K}$ (Figure 12).

Since $X$-traps are always present even in pure crystals, and since trapping processes can be so tremendously effective, it seems justified to say that one can hardly overestimate the importance of traps and trapping processes in organic molecular crystals. 
ENERGY TRAPPING PROCESSES IN AROMATIC CRYSTALS

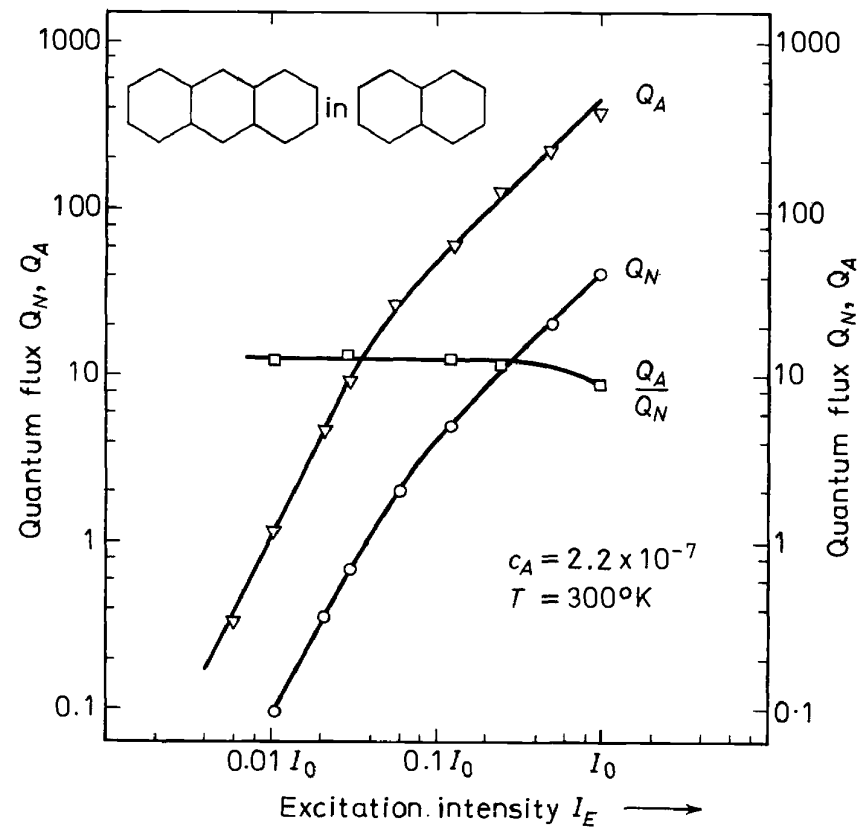

Figure 11. Quantum flux $Q_{A}$ and $Q_{N}$ and quantum ratio $Q_{A} / Q_{N}$ of delayed fluorescence in naphthalene crystals containing $2.2 \times 10^{-7}$ parts of anthracene, as a function of excitation intensity $I$, room temperature. From reference 14

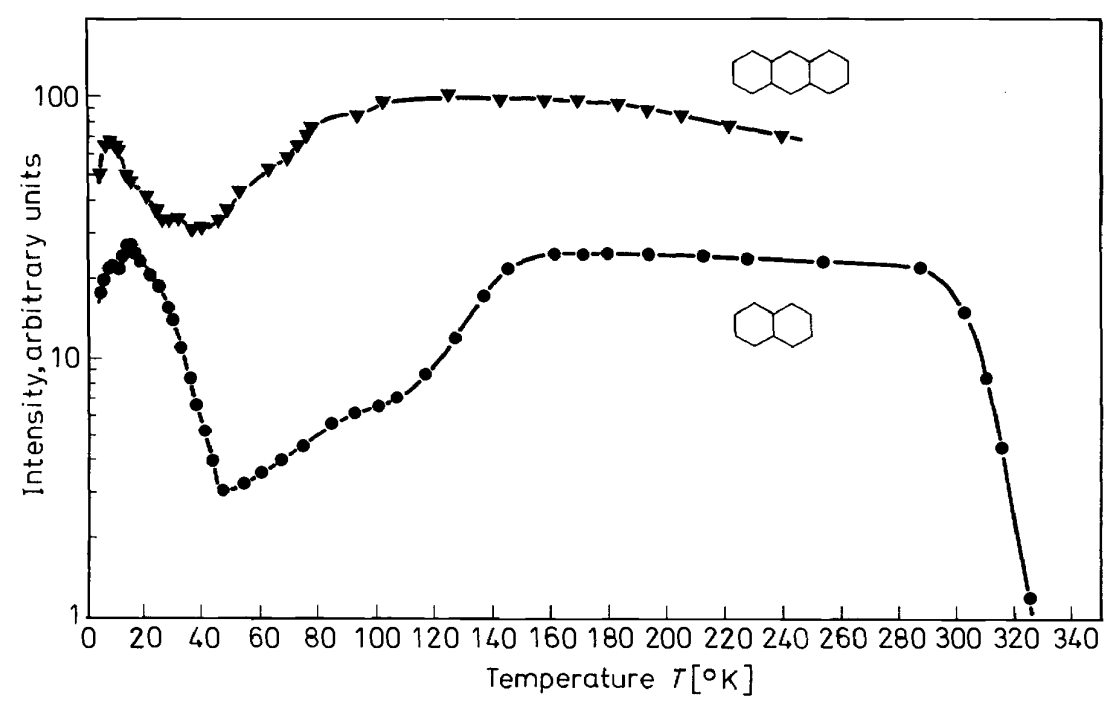

Figure 12. Integral intensity of delayed fluorescence in typical very pure anthracene and naphthalene crystals as a function of temperature. From reference 15 


\section{ACKNOWLEDGEMENT}

Figures 3, 4, 5, 8, 9 and 12 have appeared previously in Die Zeitschrift für Naturforschung and permission to reproduce them here is gratefully acknowledged.

\section{REFERENCES}

1 Y. I. Frenkel, Phys. Rev. 37, 17, 1276 (1931).

2 J. W. Sidman, Phys. Rev. 102, 96 (1956).

${ }^{3}$ H. L. Jetter and H. C. Wolf, Phys. Stat. Sol. 22, K39 (1967).

${ }^{4}$ E. Glockner and H. C. Wolf, Z. Naturforsch. 24a, 943 (1969).

5 S. A. Rice, G. C. Morris and W. L. Greer, J. Chem. Phys. 52,4279 (1970).

6 R. Ostertag and H. C. Wolf, Phys. Stat. Sol. 31, 139 (1969).

7 A. Pröpstl and H. C. Wolf, Z. Naturforsch. 18a, 724 (1963).

${ }^{8}$ See, for instance, Physics of Color Centers Ed. W. B. Fowler, Academic Press, New York (1968).

9 R. Schnaithmann and H. C. Wolf, Z. Naturforsch. 20a, 76 (1965).

10 E. Glockner and H. C. Wolf, Z. Naturforsch. 24a, 943 (1969).

${ }_{11}$ G. C. Morris et al. Fifth Molecular Crystal Symposium, Philadelphia (1970).

12 L. E. Lyons and L. J. Warren, Fifth Molecular Crystal Symposium, Philadelphia (1970).

${ }^{13}$ H. Port and H. C. Wolf, Z. Naturforsch. 23a, 315 (1968).

${ }^{14}$ K. W. Benz, Dissertation, Stuttgart (1970).

${ }^{15}$ K. W. Benz, W. Häcker and H. C. Wolf, Z. Naturforsch. 25a, 657 (1970).

16 T. Azumi and Y. Nakano, J. Chem. Phys. 51, 2515 (1969).

17 M. Schwoerer and H. Sixl, Chem. Phys. Lett. 2, 14 (1968) and 6, 21 (1970).

18 H. Sixl and M. Schwoerer, Z. Naturforsch. 25a, 1383 (1970).

19 R. E. Merrifield, J. Chem. Phys. 38, 920 (1963).

20 S. A. Rice and J. Jortner, in Physics and Chemistry of the Organic Solid State Ed. Fox, Labes and Weissberger, Vol. 3, Interscience, New York (1967).

21 J. R. Haynes, M. Lax and W. F. Flood, J. Phys. Chem. Solids 8, 392 (1959).

22 B. S. Sommer and J. Jortner, J. Chem. Phys. 50, 187, 822, 839 (1969).

${ }^{23}$ H. C. Wolf, in Advances in Atomic and Molecular Physics Ed. Bates and Estermann, Vol. 3, Academic Press, New York 1967.

24 W. Häcker, Diplomarbeit, Stuttgart (1969).

25 A. Suna, Phys. Rev. B1, 1716 (1970).

26 K. W. Benz and H. C. Wolf, Z. Naturforsch. 19a, 177 (1964).

${ }^{27}$ R. P. Groff, R. E. Merrifield and P. Avakian, Chem. Phys. Lett. 5, 168 (1970).

${ }^{28}$ A. Hammer, Dissertation, Stuttgart (1968).

29 D. Haarer and H. C. Wolf, Mol. Cryst. 10, 359 (1970).

${ }^{30}$ H. Kolb and H. C. Wolf, Colloque Ampere Bukarest (1970).

31 E. M. Yee and M. A. El Sayed, J. Chem. Phys. 52, 3075 (1970).

32 H. Baessler, G. Vaubel and H. Kallmann, J. Chem. Phys. 53, 370 (1970). 